\title{
Gottessymbol und soziale Struktur
}

\author{
Von RAGNAR HOLTE
}

Wie das Verhältnis zwischen Religion und Gesellschaft beschaffen ist, hat man in der älteren Religionssoziologie lebhaft diskutiert, wobei E. Durkheim und M. Weber zwei gegensätzliche Standpunkte vertraten. Für Durkheim waren Religion und Gesellschaft aufs Engste miteinander verwoben dergestalt, dass die Religion letzten Endes ein Produkt des kollektiven Geistes war, der den verschiedenen Gesellschaftsformen zugrunde lag. Die Religion lieferte die Kategorie der Heiligkeit und verband diese mit verschiedenen Zügen der gesellschaftlichen Struktur, die dadurch ein Gepräge der Unantastbarkeit erhielt. Die Religion dient hier also als Faktor zur Stabilisierung der gesellschaftlichen Struktur und der damit gegebenen Machtverhältnisse ${ }^{1}$. Weber dagegen betonte die relative Selbständigkeit der Religion gegenüber der sozialen Struktur, weshalb man die erste nicht einfach als ein Produkt der letzteren auffassen dürfe. Dagegen konnten religiöse Ideen - ebenso wie andere aus dem menschlichen Geist hervorgegangene Ideen - das äussere Geschehen beeinflussen und gestalten und damit auch zur Veränderung der gesellschaftlichen Strukturen beitragen ${ }^{2}$.

Das Problem, mit dem Durkheim und Weber rangen und das sie auf verschiedene Weise zu lösen versuchten, ist heute noch ebenso aktuell und dringlich und kann keinesfalls als fertig durchdacht gelten. In der wissenschaftlichen Diskussion der Folgezeit dürfte Webers Anschauung es wesentlich schwerer gehabt haben, sich zu behaupten, als die Durkheims. Webers Auffassung schien allzu eng mit dem heute verlassenen Standpunkt der idealistischen Philosophie und Geschichtswissenschaft verknüpft. Auch war es nicht schwer, zahllose Belege für die These Durkheims von der Religion als einem gesellschaftlich stabilisierenden Faktor beizubringen. Dennoch hat man auch gegen Durkheims These Einwände erhoben. Seine Antwort erwies sich als allzu eindeutig und einseitig, um ein getreues Bild der vielschichtigen Frage Religion und Gesellschaft zu geben. Man hat $u$. a. auf jene Fälle hingewiesen, in denen religiöse Ideen tatsächlich und nachweisbar zu Veränderungen der sozialen Struktur beigetragen oder den Anstoss dazu gegeben haben ${ }^{3}$.

Mein heutiges Thema, Gottessymbol und soziale Struktur, betrachte ich als ein wichtiges Teilproblem dieses grösseren Fragenkreises. Unter Gottessymbolen verstehe ich dabei die Bilder oder die bildlichen Ausdrücke

${ }^{1}$ Cf. Durkheim $21 \mathrm{ff}$., $462 \mathrm{ff}$.

merath besonders $150 \mathrm{ff}$. Die religionsphilo-

${ }^{2}$ Cf. Weber $118 \mathrm{ff}$

${ }^{3}$ Für die spätere Diskussion siehe z. B. Desophische Seite des Problems wird u. a. von Hick, 31 ff., diskutiert. 
der Sprache, mit deren Hilfe sich die Menschen den Gott/die Götter zu veranschaulichen suchen. Dabei gehe ich - im Anschluss an die Einleitungsvorlesung H. Biezais" - von dem bipolaren Charakter der Gottessymbole aus, dass nämlich ein Unterschied zwischen dem Symbol und dem Symbolisierten besteht. Ferner fasse ich mit Biezais diese Bipolarität in erster Linie als psychologisch auf, das heisst: auch wenn wir die Grenze der ,,subjektbezogenen Wirklichkeit" nicht überschreiten, in der ,, das Subjekt in seiner psychischen Aktivität den Inhalt seines Erlebnisses durch etwas Anderes [...] indirekt ausdrückt" ${ }^{\text {"4 }}$, so müssen wir an dem prinzipiellen Unterschied zwischen Symbol und Symbolisiertem festhalten. Auf die Frage, ob man das Recht hat, den Erlebnisinhalt, der durch das Gottessymbol vermittelt werden soll, auf eine vom Subjekt unabhängige ontologische Wirklichkeit zurückzuführen, gehe ich nicht ein. Für den hier verfolgten Zweck ist die Beantwortung dieser Frage nicht notwendig, die wenigstens teilweise von ausserwissenschaftlicher Art sein dürfte.

In vielen Religionen spielt ja das plastisch geformte (in Stein gemeisselte, in Holz geschnitzte, in Erz gegossene), das auf eine Fläche eingeritzte oder das gezeichnete und gemalte Götterbild eine zentrale Rolle im religiösen Kult. Bei diesen Religionen bezöge sich unser Thema vor allem auf das Verhältnis dieser Kultbilder zur sozialen Struktur. Andere Religionen sind kritisch gegenüber solchen Kultbildern oder lehnen sie vollständig ab, aber bei der Bezeichnung Gottes oder der Anrede, mit der man sich an Gott wendet, ist ihre Sprache voll von bildhaften Ausdrücken wie Vater, Herrscher u. s. w. Hier konzentriert sich unser Thema mehr auf die Frage, wie die Wahl sprachlicher Symbole für Gott von der sozialen Struktur beeinflusst wird oder eventuell diese beeinflusst.

Die Sprache selbst ist ja ein Produkt der Gesellschaft und dient zum Ausdruck menschlicher Erfahrungen und zu ihrer Vermittlung an die Mitmenschen. Je enger Religion und Gesellschaft miteinander verwoben sind, desto natürlicher fungiert die Sprache als Ausdruck und zur Vermittlung von Erfahrungen und Glaubensvorstellungen vom Wesen Gottes/der Götter, von seinem/ihrem Handein und seinen/ihren Eigenschaften. Die religiöse Erfahrung ist dann nämlich ein Teil der gesellschaftlich bedingten menschlichen Erfahrung.

In vielen von der Religion geprägten Gesellschaftsformen haben die Gottesvorstellungen ausgesprochen antropomorphen Charakter: die dem Gott zugeschriebenen Eigenschaften unterscheiden sich prinzipiell nicht von den innerhalb der Gesellschaft als ideal-menschlich gewerteten Eigenschaften, sodass auch Menschen mit den von der Religion gepriesenen Eigenschaften als göttlich betrachtet werden können. Solche göttlichen Eigenschaften können z. B. gesellschaftlich-menschliche Züge wie Fruchtbarkeit, Machtvollkommenheit oder Jagd- und Kampftüchtigkeit sein. Diese Auffassung

${ }^{4}$ Cf. oben S. XVII. 
lässt sich nicht nur in der von Durkheim untersuchten australischen Religion nachweisen, sondern auch z. B. in der altnordischen und in der klassisch griechischen und römischen Religion.

In höher entwickelten Religionen begegnet uns oft eine deutliche Tendenz, Gott/die Götter und die religiösen Phänomene stärker zu transzendieren (wobei der Plural Götter vielleicht überflüssig ist, da dieser Grad der Transzendenz wohl den Monotheismus voraussetzen dürfte). Man stellt Gott als qualitativ vom Menschen verschieden und als über diesen erhaben dar, was aber eine intime Verknüpfung von Religion und Gesellschaft nicht zu beeinträchtigen braucht.

In der Antike steht die Religion Israels mit ihrer Tendenz zum Transzendieren der Gottheit im Gegensatz zu den Religionen benachbarter Völker. Ein Ausdruck dafür ist das Bildverbot (2. Mos. 20,4-5). Während etwa in dem von den Propheten ständig bekämpften Baalskult das gemeisselte oder geschnitzte Götterbild eine zentrale Rolle spielte, gab es in Salomos Tempel in Jerusalem zwar die Abbildung der vier Engel, die den Cherubenthron Gottes trugen, Jahwe selbst aber wurde nicht abgebildet, man stellte sich ihn unsichtbar thronend vor. Eine strikte örtliche Festlegung Gottes auf den Tempel lehnt man in Israel ausserdem durch die dem König Salomo zugeschriebenen Worte ab: Siehe der Himmel und aller Himmel Himmel können dich nicht fassen - wie soll es denn dies Haus tun, das ich gebaut habe? (1. Kön. 8,27) Der leere Cherubenthron symbolisiert aber höchst greifbar die Gegenwart einer unsichtbaren, prinzipiell allgegenwärtigen Gottheit. Durch eben diese Eigenschaften stellt sich die Gottheit als radikal anderen Wesens und als erhaben über den Menschen und die menschliche Gemeinschaft dar. Zugleich aber vermittelt der Thron Assoziationen zu der menschlich-sozialen Funktion politischer Machtausübung. Epitheta der politischen Machtsphäre gibt das Alte Testament Gott ganz selbstverständlich bei, man nennt ihn Herrn, Herrscher, König u.s.w. Religiöse und politische Gemeinschaft fallen im alten Israel zusammen. Der irdische Herrscher steht unter dem Schutz des himmlischen Herrschers, er ist dem letzteren aber auch Rechenschaft schuldig und kann von ihm verworfen werden (das Schicksal Sauls). Das Königtum von Israel wird durch die Religion zugleich legitimiert und relativiert, man denke auch an die ständige, schneidende Kritik der Propheten an den Königen, wie sie ihre Macht missbrauchen.

Das Christentum tritt in vieler Hinsicht das Erbe Israels und seiner Gottesauffassung an, aber es bestehen auch bedeutsame Unterschiede. An den Vorstellungen von der Erhabenheit, Unsichtbarkeit und Allgegenwart Gottes hält man natürlich fest, sie werden noch dadurch verstärkt, dass seine Gegenwart nun nicht mehr an den Tempel von Jerusalem oder an einen anderen Kultplatz gebunden ist (Joh. 4,21-24). Das Verhältnis wird aber durch die Stellung der Person Jesu kompliziert. Da man sich die Gottheit in Jesus Christus inkarniert vorstellt und ihn folglich mit göttlichen 
Eigenschaften begabt denkt, eröffnen sich dem Christentum trotz allem bessere Möglichkeiten als dem Judentum, das Wesen Gottes durch die bildende Kunst zu veranschaulichen.

Das Christentum lehrt ja die Dreieinigkeit Gottes. Das alttestamentliche Bildverbot hat man im Verlauf der Kirchengeschichte immer wieder diskutiert, wobei bildfeindliche und bildfreundliche Richtungen miteinander in Konflikt gerieten ${ }^{5}$. Im grossen Ganzen darf man sagen, dass bildliche Darstellungen Christi als legitim betrachtet wurden, sie sind also für das Christentum bezeichnend und stehen in klarem Gegensatz zum Judentum. Aber wie verhält es sich mit Gott-Vater, der ersten Person der Gottheit? Seine Darstellung lässt sich nicht durch die Inkarnationslehre begründen, die schon im ältesten Christentum die Darstellung Jesu zuliess. Vor dem 12. Jh. gibt es praktisch auch kaum Bilder, die Gott-Vater darstellen, aber von dieser Zeit an sind sie in dem immer reicheren Bildschmuck der Kirchenwände und -fenster nicht ungewöhnlich ${ }^{6}$. Diese Tendenz zur anthropomorphen Darstellung der ersten Person der Gottheit verstärkte sich deutlich, als die eher stilisierende Darstellung des Mittelalters in die Renaissancekunst mit ihrem lebhaften Interesse für den Menschen und mit ihrer Betonung des menschlichen Körpers in der Kunst übergeht. Als einen Höhepunkt dieser Entwicklung darf man vielleicht Michelangelos Gemälde in der Sixtinischen Kapelle der Peterskirche von Rom auffassen, wo er den Schöpfer Gott als älteren, bärtigen Mann darstellt, der nicht nur göttliche Macht sondern auch physische Kraft ausstrahlt. In späteren Phasen der christlichen Kunst sind wohl die Bilddarstellungen von Gott-Vater wieder seltener geworden. Im Judentum dagegen wären sie in allen Epochen auch als Ausnahme undenkbar gewesen.

Zusammenfassend muss man daher sagen, dass jene Dimension der göttlichen Transzendenz, die im Bildverbot des Alten Testaments ihren Ausdruck findet, im Christentum wesentlich gemildert ist. In anderer Hinsicht dürfte aber die Transzendierung im christlichen Bereich gewachsen sein, ich denke hier an das Verhältnis der Gottesauffassung zur sozialen Struktur, ein für unser Thema zentraler Aspekt. Mit Ausnahme von einigen kleineren Gruppen hat man im Christentum die religiöse Gemeinschaft als prinzipiell von der politisch-sozialen Gemeinschaft unterschieden und als über diese erhaben betrachtet. Womit nicht gesagt ist, dass man gemeint hat, die Religion habe keine sozialen und politischen Folgen. Während aber das alte Israel ein theokratischer Staat war und die Messiaserwartung eine ausgesprochen politische Bedeutung hatte, unterscheidet man im Christentum normalerwise zwischen dem Reich Gottes (dem Reich Christi) und den irdischen Reichen, und man erklärt, das erstere ,, sei nicht von dieser Welt" (Joh. 18,36). Dessen ungeachtet verwendet man auch im christlichen Be-

${ }^{5}$ Im 16. Jh. hat der reformierte Zweig der

${ }^{6} \mathrm{Cf}$. Sachs 153.

Reformation die Bildfeindlichkeit vertreten. 
reich politische Epitheta als Gottesnamen, und man erhebt Gott auch hier bildlich auf einen (himmlischen) Thron. Wenn auch der Thron als Kultgegenstand im Christentum nicht mehr vorkommt, so bezeichnet man doch zuweilen den Altar als den Gnadenthron.

In Religionen mit einem Kodex von für heilig gehaltenen Schriften verbindet sich die Tendenz, die Gottheit zu transzendieren, oft mit der Lehre, der Inhalt dieses Kodex sei göttliche Offenbarung. Man folgert dann gerne daraus, dass die in diesem Kodex vorkommenden Aussagen über das Wesen Gottes, über seine Eigenschaften und sein Handeln absolut und unantastbar seien. Sie sind prinzipiell über die soziale Struktur erhaben und werden von dieser nicht beeinflusst, weshalb sie sich bei Veränderungen der sozialen Struktur nicht verändern dürfen. Besser geschulte Vertreter dieser Religionen geben jedoch zu, dass Gottessymbole und soziale Strukturen tatsächlịch voneinander abhängig sind. Man gesteht offen $\mathrm{zu}$, dass die religiöse Sprache ihre Terminologie weitgehend von gewöhnlichen menschlichen und sozialen Zusammenhängen ableitet. Wie diese Begriffe als Aussagen über Gott gebraucht werden können, erklärt man mit einer Symboltheorie, wie sie im christlichen Bereich schon in der Urkirche und im Mittelalter entwickelt wurden (z.B. Augustinus und Thomas von Aquino) ${ }^{7}$.

Im Christentum ist in diesem Zusammenhang der Gedanke der Ebenbildlichkeit von entscheidender Bedeutung. Wenn der Mensch zum Ebenbild Gottes erschaffen ist (1. Mos. 1,27), erscheint es natürlich, dass die Aussagen über diesen Gott teils aus Erfahrungen des Menschen und der menschlichen Gemeinschaft stammen und teils moralische Implikationen enthalten, wie die Menschen leben sollen und die menschliche Gemeinschaft aussehen soll. Wenn die sozialen Verhältnisse sich verändern und die moralischen Erfahrungen und Einsichten der Menschen sich entsprechend wandeln, während die in einer heiligen Schrift niedergelegten Gottessymbole als unveränderlich betrachtet werden, entsteht ein Konflikt. Im Folgenden werde ich diesen Konflikt im christlichen Bereich untersuchen, da die christliche Religion mein Forschungsgebiet ist. Ähnliche Konflikte lassen sich aber sicher auch in anderen Religionen nachweisen.

Um auf den anfangs berührten Gegensatz zwischen Durkheim und Weber zurückzukommen, möchte ich betonen, dass man sich vor zwei Extremen hüten muss. Es lässt sich nicht leugnen, dass die Religion, also auch das Christentum, oftmals die herrschende soziale und politische Ordnung religiös legitimiert hat. Dagegen hat sie in anderen Zusammenhängen auch den Anstoss zur Kritik an dieser Ordnung und zu Bemühungen um soziale Reformen und zuweilen sogar zur Revolution gegeben. Im letzteren Falle ist es aber sicher nicht so zugegangen, dass christliche Menschen unabhängig von sozialen und politischen Zusammenhängen bestimmte religiöse

7 Für Augustinus siehe Holte $329 \mathrm{ff}$. Für

Thomas siehe Lyttkens $244 \mathrm{ff}$. 
Ideen konzipiert haben, die sie dann sekundär auf die soziale und politische Wirklichkeit angewendet haben. In beiden genannten Funktionen spiegelt das Christentum soziale Erfahrungen, aber Erfahrungen verschiedener Art.

Als das Christentum in die Welgeschichte eintrat, fehlten ihm alle Verbindungen mit den religiösen und politischen Instanzen, in dem besetzten Israel so gut wie im römischen Staat. Seine Anhängerschaft bestand fast ausschliesslich aus Menschen ohne religiöse, wirtschaftliche oder politische Macht. Das Christentum als eine Religion der Armen wird in manchen Abschnitten des Neuen Testaments besonders betont, vor allem in der Evangelientradition des Lukas und im Jakobusbrief. Diese Lage änderte sich radikal, als das Christentum unter Konstantin zu einer anerkannten Religion im römischen Reich wurde, um dann zur Staatsreligion erhöht zu werden. In der früheren Situation trug das Christentum dazu bei, bei den Menschen, die sozial unterlegen waren, das Selbstgefühl und das Bewusstsein ihrer Menschenwürde zu stärken. Im späteren Falle erhielt es solche Formen, dass es das Vakuum, das die zerfallende römische Staatsreligion hinterliess, auszufüllen vermochte. In beiden Fällen handelt es sich um soziale Erfahrungen und Bedürfnisse: einerseits das Bedürfnis der Ausgestossenen und Verachteten, elementaren Respekt und Anerkennung zu erlangen, und die Erfahrung, dass der Glaube an einen Gott, der diejenigen liebt und auserwählt, die im Auge der Welt nichts sind, das ermöglicht (1. Kor. 1,26-28). Andererseits das Bedürfnis der politischen Macht, ihre Stellung göttlich zu legitimieren, und die Erfahrung, dass dies mit Hilfe des Christentums geschehen kann, sofern man andere Züge des Neuen Testaments hervorhebt. Der römische Staatsbürger Paulus hatte ja gelehrt, dass die Obrigkeit von Gott eingesetzt sei (Röm. 13), und er hatte auch sonst einer Über- und Unterordnung das Wort geredet (zwischen Herren und Dienern, zwischen Mann und Frau u. s. w.: 1. Kor. 11, Eph. 5-6).

Man kann daher sagen, dass das Christentum durch seine gesamte Geschichte hindurch sozial betrachtet zwei verschiedene Gesichter aufweist, und dass es sich in beiden Gestalten an Hand der Bibel legitimiert hat. Im Mittelpunkt der Botschaft des Neuen Testaments steht die Verkündigung der Einheit der ganzen Menschheit, der Gleichwertigkeit aller Menschen als Geschöpfe Gottes, die von der Schöpfungsabsicht Gottes abgefallen und durch Christus erlöst sind. In diesem Sinne kann Paulus sagen: „Hier ist nicht Jude noch Grieche, hier ist nicht Knecht noch Freier, hier ist nicht Mann noch Weib; denn ihr seid allzumal einer in Christus Jesus" (Gal. 3,28). Daneben gibt es aber im Neuen Testament (nicht zuletzt bei Paulus) Stellen, welche die Fortdauer der patriarchalischen Ordnungen vorauszusetzen scheinen (siehe oben).

Wie lässt sich das vereinbaren? Schon die Theologen der alten Kirche erkannten klar die Spannung zwischen der zentral religiös motivierten Verkündigung der Gleichheit im Evangelium und der dort vorausgesetzten 
patriarchalischen Struktur der Gesellschaft. In der Regel deutete man diese Spannung so, dass eine Gesellschaft, in der die Menschen gleichberechtigt nebeneinander leben, der eigentlichen Schöpfungsabsicht Gottes entspreche - und damit einer idealen Ethik. Mit einer vom Stoizismus entliehenen Terminologie sprach man von einem absoluten Naturrecht. Die Struktur der Über- und Unterordnung mit einer gewissen daraus folgenden Zwangsmacht ergab sich dagegen zwangsläufig aus dem Abfall der Menschen von Gottes Schöpfungsplan, der dazu führte, dass sie egoistisch nach Macht und eigenem Vorteil auf Kosten anderer strebten. Nach stoischer Terminologie sprach man hier von einem relativen Naturrecht $t^{8}$.

Der Ethik, die den sozialen Ordnungen zugrunde liegt, erkennt man also nur relative Gültigkeit zu. Die ideale Ethik dagegen zeigt, wie die Menschen eigentlich zusammenleben sollten, und Jesus hat sie den Menschen in seiner ethischen Belehrung (z.B. in der Bergpredigt und in seiner Verkündigung des Reiches Gottes) erneut vorgestellt. Diese ist in religiöser Hinsicht - vor Gott - absolut gültig.

Müsste sich dann nicht die ideale Ethik wenigstens nach und nach durchsetzen und auch die sozialen Verhältnisse prägen? Die Beantwortung dieser Frage zeigt das Christentum, wie es sich im Verlauf der Geschichte entfaltet, von zwei ganz verschiedenen Seiten. Die Antworten weisen nämlich in zwei verschiedenen Richtungen.

Eine Richtung der Tradition, für die sich die anerkannten Kirchen häufig entschieden haben, betrachtet die ideale Ethik als ausschliesslich auf das geistliche Gebiet beschränkt. Die sozialen Strukturen, von denen in der Bibel die Rede ist, sind unauflöslich mit den Lebensbedingungen dieser Erde verbunden und müssen bis zum jüngsten Tag bestehen ${ }^{9}$. Damit weicht man also hier von der in der frühchristlichen Kirche insgemein vertretenen Auffassung ab, dass die gesellschaftliche Ordnung ethisch nur relativ gültig ist und der eigentlichen Absicht Gottes mit der Schöpfung nicht entspricht. Die gesellschaftliche Rangordnung hat man innerhalb dieser Tradition bisweilen sogar als eine unveränderliche Ordnung der Schöpfung bezeichnet $^{10}$.

Die entgegengesetzte Traditionsrichtung sieht im Evangelium eine Botschaft der Befreiung der Bedrückten, der Überwindung der destruktiven Kräfte, die das Menschenleben verheeren, sie meint daher, dies müsse auch Folgen für die sozialen Bedingungen der Menschen haben. Man muss

\footnotetext{
${ }^{8}$ Für die historische Entfaltung des Verhältnisses Christentum - soziale Struktur muss man immer noch den Einsatz von Troeltsch hervorheben. Er hat auch die Bedeutung der Unterscheidung zwischen absolutem und relativem Naturrecht bei den Kirchenvätern betont. Cf. $144 \mathrm{ff}$. Für Augustinus siehe z. B. De civitate Dei XIX,14ff. (CC 48). Die Unterordnung der Frauen führt er jedoch, von
}

dieser Grundauffassung abweichend, auf Gottes Schöpferwille zurück: De Genesi ad litt. XI,35 (CSEL 28).

9 Diese Auffassung hat u. a. Luther vertreten. Cf. Luther $11,251 \mathrm{ff}$. Cf. Duchrow $486 \mathrm{ff}$. ${ }^{10}$ So z. B. Brunner $313 \mathrm{ff}$. Von dieser Auffassung nehmen so gut wie alle moderne theologische Ethiker Abstand. 
die altchristliche Einsicht, dass die soziale Rangordnung ethisch nur bedingt gültig ist, ernst nehmen, sie muss im Licht der idealen Ethik kritisiert und nach Möglichkeit in bessere Übereinstimmung mit dieser gebracht werden.

Träger dieser Auffassung waren oft religiöse Minoritäten, welche die anerkannten Kirchen kritisierten, man denke etwa an die radikalen Strömungen der Reformationszeit oder an Gruppen der Auswanderer nach Nordamerika im 18. und 19. Jh. Aber auch in der institutionalisierten Kirche wirkt sich diese Kraft aus, - wenn auch in langsamerem Takt. So gab z.B. die christliche Kirche den Anstoss zur Abschaffung der Sklaverei. Hier war Schweden übrigens seiner Zeit voraus: zu Anfang des 14. Jhs. wurde die Sklaverei sukzessiv in allen Provinzen abgeschafft, - mit einer in unserem Zusammenhang bedeutsamen Begründung: Da Christus alle Menschen durch die Taufe freigekauft habe, dürfe niemand als Knecht verkauft werden ${ }^{11}$.

Die Lebenskraft einer Religion dürfte in hohem Masse davon abhängen, ob sie den Menschen zu einer existenziellen Deutung ihrer Lebenslage verhelfen kann. Die Gotteserfahrung muss mit einer Ich-Erfahrung verbunden sein, die das Selbstbewusstsein und das Erlebnis der eigenen Menschenwürde unterstützt. Das kann aber unmöglich jenseits der gesellschaftlichen Zusammenhänge geschehen. Die Gotteserfahrung muss dem Menschen auch dazu verhelfen, seine soziale Rolle zu finden und sie muss ihm die Identifikation mit anderen Menschen im sozialen Rahmen ermöglichen. Wenn die Religion und ihre Symbole mit einer gesellschaftlichen Struktur verbunden sind, die sich nicht mehr als Rahmen der eigenen sozialen Situation deuten lässt, so kann die Religion vielleicht noch eine Zeitlang kraft ihrer äusseren Glaubensautorität weiterleben, aber auf längere Sicht ist sie zum Absterben verurteilt. Dies dürfte einer der wichtigsten Gründe sein, weshalb der Weg des Religionshistorikers - um wiederum Biezais' Einleitungsvortrag zu zitieren - weitgehend ,,ein Spaziergang auf dem Friedhof der toten religiösen Symbole ist" ${ }^{612}$. ,Symbole, die einstmals Ausdruck tiefer religiöser Erlebnisse waren, haben im Lauf der Zeit ihren Sinn verloren und sind deshalb unverständlich geworden." Wir können hinzufügen: auch Symbole, in die wir uns mit unserer Phantasie hineindenken können, haben dennoch vielfach ihre existenzielle Funktion für uns verloren.

Durch den im 18. Jh. einsetzenden Prozess der Demokratisierung, der die Gesellschaftsstruktur in der ganzen traditionell christlichen Welt immer durchgreifender gewandelt hat, ist für das Christentum in dieser Hinsicht eine radikale Veränderung eingetreten. Dabei ist es übrigens charakteri-

${ }^{11}$ Dass in anderen Ländern die Kirchen auch viel später die Sklaverei bisweilen unterstützt haben, stimmt damit gut überein, was obr über den zwei verschiedenen Gesichtern des Christentums gesagt wurde.

12 Oben S. XXV1. 
stisch, dass das Christentum in so gut wie allen Phasen dieses Demokratisierungsprozesses sein doppeltes Gesicht gezeigt hat. Christen die von der Verkündigung der Gleichheit vor Gott im Neuen Testament ausgingen, haben die Meinung vertreten, dass diese auch im Staatswesen Folgen haben müsse, und deshalb den Prozess der Demokratisierung unterstützt. Andere Christen, die sich auf die patriarchalischen Züge des Neuen Testaments stützten, haben die Gleichheit auf das religiöse Gebiet begrenzen wollen und folglich der Demokratisierung entgegengearbeitet.

Auf Grund des Demokratisierungsprozesses hat sich unser Problem folgendermassen verändert: Vor dem Beginn desselben war die Religion oft die einzige Möglichkeit für die Menschen, einen politischen Protest zu äussern, den man aber nicht direkt und klar aussprach, sondern der indirekt darin zum Ausdruck kam, dass man sich die religiöse Umwertung weltlicher Machtverhältnisse zu eigen machte und aus dem Glauben, von Gott geliebt zu sein, ein erhöhtes Selbstgefühl gewann. Dies Bewusstsein in einen direkten sozialen Prozess umzusetzen, hätte vielfach bedeutet, sich der repressiven Gewalt des patriarchalischen Staates unmittelbar auszusetzen. Unter diesen Umständen konnte sich der Protest nicht in Form einer ausdrücklichen Forderung von sozialen Reformen äussern, sondern er hat den Menschen vor allem die Kraft gegeben, dem Druck der sozialen und politischen Verhältnisse standzuhalten. Seit dem Beginn des Demokratisierungsprozesses ist diese Maskierung des sozialen Protestes bei weitem nicht mehr im gleichen Masse erforderlich, - jetzt fordert man soziale und politische Reformen direkt.

Dieser Übergang vom indirekten zum direkten sozialen Protest zeigt sich sehr deutlich bei der Auswanderung nach Nordamerika im 18. und 19. Jh. Unter den Auswanderern gab es viele, deren Religiosität mit der Ablehnung der autoritären politischen Systeme in ihren Heimatländern verbunden war. Dort hatten sie im allgemeinen nicht die Möglichkeit gehabt, ihren Protest offen auszusprechen, was sie in der neuen Heimat aber taten. Hier wollte man einen Staat gründen, in dem der vollkommene Menschenwert aller gemäss der Gleichheitsverkündigung des Evangeliums verwirklicht werden sollte. Die neu zu begründende Demokratie sollte, wie man es zuweilen etwas naiv ausdrückte, das Land zum „,Kingdom of God in America" machen ${ }^{13}$.

Ein bezeichnendes Beispiel aus unserem Jahrhundert liefert der Freiheitskampf der Neger in den U.S.A. Paradoxalerweise gab es ja in dem Staat, der sich prinzipiell auf den vollkommenen Menschenwert und die Gleichberechtigung aller gründete, eine umfangreiche Gesetzgebung zur Segregation. Widersetzte man sich dieser Gesetzgebung - sei es in Reden oder durch Handlungen - so löste dies die repressive Gewalt des Staates

13 Cf. das Buch von Niebuhr, besonders $33 \mathrm{ff}$. Für das Verhältnis Christentum - soziale
Struktur seit dem 19. Jh., siehe Latourette, besonders 1, $207 \mathrm{ff}$; 3, $200 \mathrm{ff}$.; 5, $515 \mathrm{ff}$. 
aus, weshalb es lange dauerte, ehe die Schwarzen es wagen konnten, offen ihr volles demokratisches Recht zu fordern. Der soziale Protest war zunächst maskiert, lässt sich aber in der religiösen Formulierung leicht erkennen. Die negro spirituals der Schwarzen handeln fast durchweg von einem Thema: von der Knechtschaft des alttestamentarischen Gottesvolkes in Ägypten und von seiner Befreiung. Man denkt dabei natürlich an das eigene Volk und seine Lebensbedingungen in einem von Weissen beherrschten Land, und das Volk weiss sehr wohl, dass es überhaupt nur durch den Sklavenhandel nach Amerika gekommen ist. Erst in einer späteren Phase der Befreiungstheologie sprechen die Schwarzen ausdrücklich vom eigenen Volk und dessen Forderung an den amerikanischen Staat und sie bringen das weitgehend von der Religion getragene Selbstbewusstsein durch Devisen wie ,,Black is beautiful" zum Ausdruck ${ }^{14}$.

Inwiefern betrifft nun die Veränderung der sozialen Struktur die Gottessymbole selbst? Wie schon gesagt, kommen in den biblischen Texten weitgehend Gottessymbole aus der sozialen und politischen Sphäre vor, die wegen ihres Ursprungs in den sozialen und politischen Strukturen ihrer Entstehungszeit patriarchalische Assoziationen erwecken. Die Symbole stammen z. B. aus der Späre des politischen Alleinherrschers und seiner Stellung gegenüber den Untertanen: Gott ist der Herr (kýrios), der König (basileús), der Allherrscher (pantokrátor). Hierzu sei bemerkt, dass die Bezeichnung Gottes als Allherrscher, als allmächtig, nicht philosophisch zu deuten ist (Gott als erste Ursache aller Dinge u. dgl.), wie man es später oft getan hat, der Ausdruck stammt vielmehr von dem Traum des irdischen Herrschers, sein Reich über die ganze bewohnte Erde ausdehnen zu können. Bei dem Namen ,Herr" stellen sich ausserdem Assoziationen zu engeren sozialen Strukturen ein, bei denen der Hausvater oder der Vormann im Verhältnis zu den ihm untergebenen Dienern oder Sklaven (doúloi) gemeint ist. Gott wird schliesslich als Vater bezeichnet, mit Assoziationen zur Struktur der Familie und dem Verhältnis Eltern - Kinder, aber auch weitere soziale Strukturen klingen an: den Titel ,Vater" hat man vielfach auch auf politische Herrscher auf verschiedenem Niveau übertragen. In den Jesus-Worten der Evangelien dominiert das Epithet Vater (patér) als Bezeichung Gottes, im Verhältnis zu ihm ist Jesus der Sohn im wahrsten Sinne, wenn auch alle, die zum Volke Gottes gehören, seine Kinder oder Söhne (hyioí) sind. Jesus Christus wird nie der Name Vater beigelegt, sonst kann aber Christus in der christlichen Überlieferung bezeichnenderweise so gut wie alle Epitheta Gottes erhalten. Ansätze dazu liegen schon im Neuen Testament vor. Christus ist ausserdem der Bräutigam im Verhältnis zu seinen Anhängern, welche die Braut sind. Im Alten Testament

${ }^{14}$ Cf. z. B Cone $25 \mathrm{ff}$. Martin Luther King war wohl der erste, der den sozialen Protest der Schwarzen offen aussprach. Die mo- derne ,Black theology“ tut das aber in einer bedeutend aggressiveren Weise. 
war dies Symbol Jahwe im Verhältnis zu seinem Volke Israel vorbehalten. Man muss beachten, dass auch dies Symbol einen patriarchalischen Anklang hat, da in ihm die Unterordnung der Ehefrau gegenüber ihrem Mann vorausgesetzt wird.

Nun mag es zunächst erscheinen, dass der Gebrauch von Gottessymbolen dieser Art in doppeltem Sinne wirken muss: teils formt er die Haltung des Gläubigen Gott/Christus gegenüber nach dem Modell des Verhältnisses von Untergebenen/Unterdrückten zu Vätern und Obrigkeitspersonen verschiedener Art, teils gibt sie der Stellung dieser Väter und Obrigkeitspersonen eine religiöse Legitimation. Dann übersieht man aber einen Kernpunkt in der Verkündigung Jesu, wie er in den Evangelien und den auf sie gegründeten Episteln hervortritt: nämlich die totale Umwertung und Umkehrung der im gewöhnlichen Leben gültigen Rangordnung. Die Ordnung im Reiche Gottes wird hier als der diametrale Gegensatz der in sozialen und politischen Zusammenhängen gültigen dargestellt. Was die Welt gering schätzt und verachtet, hat Gott erwählt (Luk. 1,51-53; 1. Kor. 1,26-28). Nicht der ist der Grösste, der als Herr über die anderen auftritt, sondern der sich zum Diener und Sklaven anderer macht (Luk. 9,46-48; 22,24-26). In diesen Kategorien deutet Jesus auch sein eigenes Werk, und wer ihm folgen will, muss sich diesen Bedingungen beugen (Luk. 22,27; Joh. 13,1-15; Phil. 2,1-11). Gerade diese Hauptlinie der Evangelien ist es ja, die einem bald maskierten, bald offenen sozialen Protest als Inspirationsquelle hat dienen können. Psychologisch gesehen dienen hier die Machtsymbole denjenigen Gruppen als Schutz und Legitimation, denen im sozialen Leben dieser Schutz und diese Legitimation am meisten abging.

Auch für die Gottessymbole ergibt sich aus dem Prozess der Demokratisierung eine durchgreifende Veränderung. Der entscheidende Punkt, der darin lag, dass die Gottessymbole weitgehend aus der politisch und sozial gültigen Struktur stammten, aber zugleich so gebraucht wurden, dass sie diese von innen her auflösten, wird jetzt nicht mehr wie früher evident. Zugleich gerät auch jene Tradition der Auslegung, welche die Umwertung der Machtstruktur auf streng religiöse Zusammenhänge begrenzen wollte, in eine eigentümliche Lage. Zuweilen wirkt sich das so aus, dass man im Namen der Bibeltreue zu Positionen zurückzukehren strebt, die in sozialen und politischen Zusammenhängen bereits verlassen sind. So kann es passieren, dass man mit einer eigentümlichen Verdrehung des Gegensatzes, wie es in dieser Welt zugeht und wie im Reiche Christi, zwar die Demokratisierung auf weltlichem Gebiet akzeptiert, ihr aber in christlichen Zusammenhängen entgegenarbeitet, z.B. sollen Frauen zu weltlichen Berufen durchaus kompetent sein, nicht aber zum Amt des Pfarrers!

Wie schon gesagt: wenn die Religion und ihre Symbole an eine soziale Struktur gebunden sind, die man nicht mehr als Rahmen der eigenen sozialen Situation zu deuten vermag, sind die Voraussetzungen für einen persönlich fundierten Glauben nicht gegeben. Die Symbole sterben ab und 
verlieren ihren Wert. Einer der Gründe der zunehmenden Säkularisierung in traditionell christlichen Ländern mag darin liegen, dass das Christentum von dem Einzelnen nicht als persönlich relevant erlebt wird, weil seine Symbolsprache mit veralteten Strukturen verknüpft zu sein scheint.

Zugleich arbeiten im Christentum wie in anderen lebenden Religionen Individuen und Gruppen an einer Neudeutung, die neue Wege zu einem für die Persönlichkeit wesentlichen christlichen Glauben zeigt. Diese Neudeutung hat zuweilen zu herausfordernden Formulierungen geführt, wenn man z. B. in einer amerikanischen Religionsdebatte von Gott behauptet hat „,she is black". Da gibt es natürlich - wie immer - an die Tradition gebundenen Individuen und Gruppen, die solche Tendenzen sofort als Irrlehren anprangern. Dabei übersieht man leicht, dass bei dieser Kombination von , black theology" und ,,feminist theology" etwas geschieht, was in anderen Formen schon oft in der christlichen Tradition vorgekommen ist. Dass man die Gottessymbole, sowie die Symbolwelt der Bibel im Ganzen mit den Erfahrungen des eigenen Volkes, der Gesellschaftsklasse, des eigenen Geschlechts und der eigenen Person verschmelzen kann, hat sich im Verlauf der Geschichte als die Voraussetzung für die Existenz eines lebendigen, persönlich fundierten christlichen Glaubens erwiesen. Man denke nur an die Volkskunst in Dalekarlien und in anderen schwedischen Provinzen, wo Gott und die biblischen Gestalten im Milieu des schwedischen Landvolkes auftreten, oder an das Altarbild des Domes von Linköping, auf dem Jesus als blonder nordischer Jüngling dargestellt ist. Ein anderes Beispiel sind die sechs modernen Pietà-Skulpturen in der neu erbauten Kirche von Nowa Huta bei Krakau in Polen, wo die Mutter Gottes, die ihren toten Sohn beweint, durch Namen wie Auschwitz, Maidanek und Katyn mit sechs tragischen Ereignissen aus der Geschichte des politisch unterdrückten und leidenden polnischen Volkes verknüpft sind.

Man braucht die Vertreter der black, bzw. feminist theology ja nicht so zu deuten, dass sie den bipolaren Charakter der Gottessymbole aufheben wollen. Es geht wohl nicht darum zu behaupten, dass Gott tatsächlich schwarz und weiblichen Geschlechts ist, sondern darum, der Tendenz entgegenzuwirken, dass man sich Gott als einen Mann von weisser Hautfarbe vorstellt ${ }^{15}$. Denken wir doch nur an die Bilder Michelangelos in der sixtinischen Kapelle. Wenn auch diese extrem antropomorphe Darstellung Gott Vaters zu den Ausnahmen in der christlichen Tradition gehören mag, so kommt sie doch den Vorstellungen im christlichen Abendland recht nahe. Wieviele Kinder - und Erwachsene - haben sich Gott nicht als den bärtigen alten Mann, natürlich von weisser Hautfarbe vorgestellt! Zugleich ist man sich aber bei einer verantwortungsbewussten Auslegung der christlichen Religion stets darüber einig gewesen, dass ein Unterschied zwischen

${ }^{15}$ Für die ,Feminist theology“" siehe besonders Mary Dalys Programmschrift, $13 \mathrm{ff}$. 
dem Symbol und dem Symbolisierten besteht. Ihre Lehre war im Grunde immer, dass Gott in Wahrheit weder Mann noch Frau, weder schwarz-, rot- noch weisshäutig ist, vielmehr ist er ein Gott für alle, erhaben über alle menschlichen und sozialen Unterschiede, und doch zugleich allgegenwärtig und daher beiderseits aller menschlichen Schranken erlebbar.

Bewegungen wie ,,black theology" und ,feminist theology" mögen uns ferner daran erinnern, dass es auch in demokratischen Ländern Individuen und Gruppen gibt, die sich unterdrückt fühlen und denen die Möglichkeit fehlt, ihre Menschenrechte voll auszuüben. Wenn diese ihre Erfahrungen mit einer Neudeutung des christlichen Glaubens verschmelzen, so erkennt man das Muster aus früheren Phasen der Konfrontation der christlichen Tradition mit neuerwachten demokratischen Bestrebungen leicht wieder. Einerseits wird die Notwendigkeit betont, mit den in der Bibel vorausgesetzten sozialen Strukturen zu brechen, und die Gefahr erkannt, dass die politisch-patriarchalischen Gottessymbole der Bibel einer patriarchalischen Gesellschaftsordnung als Stütze dienen können. Zugleich erhält man die Inspiration zu dieser Neudeutung aus der Art, wie die Bibel selbst trotz der Anwendung dieser Symbole, die von diesen gemeinte Rangordnung umbewertet und in ihr Gegenteil verkehrt.

Eigentlich ist es erstaunlich, dass die Probleme, die mit dem Überwiegen der männlichen Symbolik in der traditionell-christlichen Weise, von Gott zu sprechen, verbunden sind, erst so spät in der geschichtlichen Entwicklung zu Worte kommen. Dasselbe gilt von der Tatsache, dass christliche Theologie und Predigt durch die Jahrhunderte fast immer Sache der Männer war. Während der ganzen Zeit hat aber die Gotteserfahrung der Frauen eine entscheidende Rolle für die Weitergabe des Glaubens an neue Generationen gespielt, man denke nur an die christliche Erziehung der Kinder! Es würde sich lohnen, die sicher recht zahlreichen schriftlichen Zeugnisse vom Gotteserlebnis christlicher Frauen zu sammeln und zu untersuchen, ob es zu dem heute offen ausgesprochenen Protest der feminist theology einen entsprechenden, weniger offenen Protest in früheren Generationen christlicher Frauen gegeben hat.

Zum Schluss muss ich betonen, dass natürlich nicht alle Gottessymbole der christlichen Tradition, die von der Bibel ausgeht, mit der sozialen Struktur verknüpft sind, und dass selbst unter diesen nicht alle patriarchalische Verhältnisse der Über- und Unterordnung betonen. Symbole wie Licht, Lebensspender, Liebe u. s. w. knüpfen an allgemein-menschliche Erfahrungen an, die durch alle sozialen Veränderungen hindurch bestehen. Die Auffassung Jesu als Bruder und Mitmensch knüpft an die Idee der menschlichen Gleichwertigkeit an. Auch hat das Vorwiegen der männlichen Symbolik in der Gottesauffassung das Vorkommen von Muttersymbolen nicht verhindert u.s.w. Die christliche Überlieferung enthält in der Tat eine Mannigfaltigkeit von Gottessymbolen, die aus den verschiedensten Bereichen menschlicher Erfahrung stammen. Wie bei anderen lebenden 
Religionen besteht das Problem darin, wie man in der Vielfalt der Symbole wählen soll, welche traditionellen Symbole hervorzuheben sind, welche man dämpfen oder fallen lassen sollte, und welchen Raum man der Neuschaffung von Symbolen gewähren soll. Mit diesen Fragen befasst sich vor allem die normative Theologie, die sich in allen lebendigen Religionen entwickelt. Die Religionswissenschaft beschränkt sich darauf zu untersuchen, wie dies geschieht, und festzustellen, dass die Arbeit an den Gottessymbolen eine Voraussetzung für das Weiterleben der Religion ist, um einen persönlich fundierten religiösen Glauben zu ermöglichen.

\section{Literaturverzeichnis}

Brunner, E., 1932, Das Gebot und die Ordnungen. Tübingen.

Cone, J., 1969, Black theology and black power. New York.

Daly, Mary, 1973, Beyond God the Father. Boston.

Demerath, N., Hammond, Ph., 1969, Religion in social context. New York.

Duchrow, U., 1970, Christenheit und Weltverantwortung. Stuttgart.

Durkheim, E., 1965, The elementary forms of the religious life. London.

Hick, J., 1963, Philosophy of religion. Englewood Cliffs.

Holte, R., 1962, Béatitude et sagesse. Paris.

Latourette, K., $1958 \mathrm{f}$., Christianity in a revolutionary age, 1-5. New York.

Luther, M., 1938, Von weltlicher Oberkeit. Werke 11. Weimar.

Lyttkens, H., 1952, The analogy between God and the World. Diss. Uppsala.

Niebuhr, R., 1937, The kingdom of God in America. Chicago.

Sachs, H., Badstübner, Neumann, H., 1973, Christliche Ikonographie in Stichworten. Berlin.

Troeltsch, E., 1912, Die Soziallehren der christlichen Kirchen und Gruppen. Gesammelte Schriften 1. Tübingen.

Weber, M., 1964, The sociology of religion. London. 